\section{Clinical decision-making: coping with uncertainty}

\section{A F West, R R West}

\section{Despite increasing medical knowledge uncertainty will always remain}

\section{SUMMARY}

Reliable research and audit information are essential to increasing medical knowledge and improving health service delivery. However there are limits to available information in terms of quality, reliability, and applicability. Furthermore, however much information is gathered, there will always be a degree of uncertainty at the point of making clinical decisions with individual patients. Unrealistic lay and professional expectations of the efficacy of information and that certainty is achievable, may be altering the traditional clinician-patient relationship. One therapeutic role of a clinician is containing the anxieties aroused in the context of uncertainty, and this role may be becoming more difficult. Reliance on protocols and fear of reprimand may lead to clinicians, in some areas of medical care, abandoning their patients at a time of need.

\section{INTRODUCTION}

Many acknowledge that there has been an information explosion in the health services over the last few decades. Vastly increased volumes of information are gathered in pursuit of knowledge and in the name of audit. The former provides the backbone of "evidence based medi-

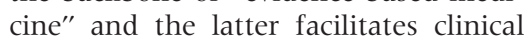
governance. $^{1-3}$ Almost certainly, more information is being gathered than will ever be put to use. The authors of this paper-one a clinician, the other an epidemiologist-view these developments from different perspectives and share a concern. They recognise the extent to which properly collected and handled research information and properly collected and handled audit information could and should improve health service delivery, ${ }^{4}$ yet are also aware of the limits to the information, both in its comprehensiveness and in its usefulness. What is more, they join to describe how the current emphasis on apparently factual information lets down both doctor and patient because it denies the importance of a crucial aspect of the relationship, namely the management of uncertainty. However much information or "evidence" there is to hand (and there will now always be more than can be satisfactorily held in mind) a decision still has to be made and, at the point of making each decision, there will always be some uncertainty.

\section{IMPORTANCE OF INFORMATION}

Few would question that a rationale exists for the "evidence based medicine" movement. Reliable information is essential to both scientific advancement and process management. This need for information has led to such quantities of evidence that clinicians need assistance in choosing which evidence should influence their practice. ${ }^{5}$

\section{LIMITS TO INFORMATION}

There are limits to the information available through research and review. ${ }^{6}$ While some areas may be over-researched, for example the surgeon general of the USA reported thousands of papers into the health effects of smoking, ${ }^{7}$ there are other areas, for example in the psychotherapies, where randomised trials and meta-analytic evidence are hard to come by and some would argue may even be inappropriate. There are also problems concerning the quality of information. Doctors are now trained to the point where they can spot a paper's more obvious shortcomings such as sampling errors, data loss, and incorrect conclusions. This is necessary because, despite a general level of awareness, important errors still slip through peer review and appear in print. Perhaps the main function of teaching medical students "critijudge a paper, but to give them a healthy scepticism and suspicion of the published word. What, then, does the nonstatistician trust as evidence? For some the meta-analysis was, for a time, perceived as the head of the "hierarchy of evidence" but it is now more widely recognised that meta-analysis is itself a sampling procedure and is subject to random effects ${ }^{9-11}$ and we are now seeing meta-analyses of meta-analyses. ${ }^{12}$ Even good systematic reviews present the clinician with questions of external validity or applicability of research findings in clinical practice. ${ }^{13}$ The certainty cal appraisal"s is not to enable them to sought by research forces researchers to simplify the measurable situation to the point where it is often unrepresentative of the typical clinical scenario: it will almost always be possible to show differences between the research samples and clinical populations, never mind the individual patient.

"Complexity science"14 may go some way towards explaining why complex systems, like people, do not behave in predictable ways, yet those authors appear to remain seduced by the search for certainty, for they say, "Ultimately the only way to know exactly what a complex system will do is to observe it". Such reductio ad absurdum illustrates a crucial point. Prediction of the future on past observations is probabilistic and is not the same as knowing exactly what will happen. Although all previous swans were white, we cannot be certain that the next will not be black. Prediction is particularly difficult when $n=1$.

\section{INFORMATION OVERLOAD}

The Yerkes-Dodson law, first propounded in 1908, describes how arousal and taskdifficulty impact on the time taken by individuals to perform at a given task; too little arousal and people cannot be bothered; too much and they becomes distracted. ${ }^{15}$ Perhaps there is a critical amount of information that a clinician can hold and which enables optimal functioning: too little and they feel uninformed; too much and they are confused with information overload. Erring in either direction could make decisionmaking harder. Certainly the curvilinear relationship between performance and levels of reward or punishment imply that, if the stakes are too high, a clinician's ability to perform at a discriminant task, such as decision-making, will fall off.

\section{THE CLINICAL DILEMMA}

The existence of an information mountain provides a myth of certainty for the patient, the public and perhaps for health care policy-makers. Certainty is an illusion. In most professional spheres the expert is more critical of the available evidence, than is the lay person. Hopefully, clinicians are taught to be more critical than patients but they are faced with a dilemma. They appear to be very well informed yet are acutely aware of the fallibility of the information that is expected to guide their practice. How do clinicians respond to this dilemma? Their day consists of a succession of points at which decisions have to be made on the basis of incomplete and inadequate information and in a context in which risk, never mind error, is outlawed.

Quick and easy solutions exist, which can be divided broadly into the clean and 
the dirty: either the responsibility is passed on to protocols and guidelines, or on to the patients themselves. Guidelines still leave the clinician the onus of making a decision and so really come into the category of "evidence". Protocols absolve the clinician who follows the protocol correctly, but facilitate criticism of the clinician who does not. They can restrict research, ironically limiting the acquisition of new evidence. They appear to restrict what action is ethical but actually remove ethical considerations from the immediate clinical decision. Finally, they threaten to depersonalise the clinical relationship to the point where the patient is effectively consulting an automaton instead of a human being.

The dirty option is to pass the responsibility on to the patient. This extreme form of user involvement has the superficial appeal of "political correctness" but is in reality an abrogation of responsibility. At least, while this form of "shared

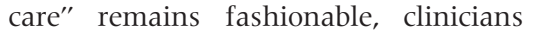
who take this route are unlikely to be criticised, but something has gone wrong with the clinical relationship (or for that matter any client-professional relationship) when clinicians quote figures, survival rates, and probabilities of side effects at their patients instead of offering them educated professional guidance and support in the difficult process of reaching a decision.

\section{THE CLINICIAN-PATIENT RELATIONSHIP}

Each of the above options may have a role but both carry a risk, particularly when applied in high doses, of undermining the relationship between the patient and the clinician. One aspect of this relationship is the creation of some belief in the process of healing. The placebo effect is mostly encountered in research and has become regarded primarily as an obstacle to be overcome. Attempts have been made to dissect its effects and explain them, for example, in behavioural terms ${ }^{16}$ and the literature acknowledges that, as well as being a bane of research, the placebo effect can be considered a boon to therapy. ${ }^{17}$ The placebo effect might be enhanced by bolstering its credibility with arcane terminology but undermining the special relationship between the clinician and the patient is likely to have the opposite effect. Another crucial function of the clinician-patient relationship is that of containing the patient's anxiety, much of which arises out of uncertainties of various kinds. To be able to do this, clinicians must be able to shoulder a substantial part of that anxiety themselves and this is only possible if they are not assailed by too many personal anxieties arising out of the clinical situation-such as summary suspension lurking round the corner. While profession-led audit may be fully justified to raise standards and reduce the incidence of errors, the current emphasis on personal error places too great a burden of personal anxiety on individual clinicians. The results are likely to include disaffection, burn-out, and a profession that seeks early retirement. The clinician requires a modicum of immunity from the consequences of mistakes, which should not be overly eroded by scrutiny, however necessary that scrutiny for malpractice or incompetence might be. Scrutiny should be focused more on systems than on individuals.

\section{INSTITUTIONALISED DEFENCES AGAINST UNCERTAINTY}

In the 1950s Isabel Menzies Lyth was asked to advise a hospital in relation to its nursing organisation. ${ }^{18}$ She and her team did so, using her very considerable psychoanalytic experience and insight. She exposed a number of mechanisms developed, largely unintentionally, as defences against the anxieties of intrinsically anxiety-provoking work. These mechanisms included denial of the significance of the individual (both patient and nurse), "the attempt to eliminate decisions by ritual taskperformance", "reducing the weight of responsibility in decision making by checks and counterchecks", and a training system that concentrated on communication of fact and technique, as though responsibility and personal maturity were innate qualities that could be taught. The result was an incomplete avoidance of the primary anxiety, a failure to support and encourage nurses in working through and mastering those anxieties, and the creation of a good deal of secondary anxiety. What is striking, on looking back over this analysis, is the extent to which the uses of information discussed in the present paper can be described in similar terms. The writing of Lyth is just one potent example of qualitative research, not easily amenable to meta-analysis. The lesson has only been partly learned.

\section{THE INEVITABILITY OF UNCERTAINTY}

Physicists have long recognised the uncertainty principal. ${ }^{19}$ Uncertainty, like anxiety, cannot be "killed". It can only be lived with, contained, or uncontained. The current attempt to "kill" uncertainty in our society risks removing from the medical profession some important "tools of the trade" and placing obstacles in their way. Too much emphasis in medical training on removing or reducing uncertainty will crowd out what little attention is being paid to educating doctors into the maturity and wisdom that they require to be able to accompany people in times of need, contain their own and their patients' anxieties and facilitate healing and recovery in an uncertain world. The public is being told, by selective publication, what to expect or demand from their doctors: factual diagnosis, when the wise doctor knows that many diagnoses are not circumscribed entities and that no tests have $100 \%$ sensitivity and specificity; factual prognosis, when the closest that can be achieved is an estimate, with a $95 \%$ confidence interval, for an average person in the population to which the patient might belong; and most effective or best available treatment, when the question "best at what?" is constantly begged and seldom satisfactorily answered.

Improving scientific knowledge is clearly a laudable objective, although it may reduce clinical uncertainty less than expected. Pretending that the clinical predicament can be reduced to a series of certainties by the recruitment of "evidence" will not work and unrealistic expectations of that stratagem may make the situation worse. Some degree of uncertainty was always here to stay and evidence, even of the highest quality, is only evidence. There will always be judgments to be made by responsible, informed, and compassionate people. They may not be able to perform these broader roles, in communication, holding anxieties and managing uncertainty, unless trained for and supported in them.

Postgrad Med J 2002;78:319-321

\section{Authors' affiliations}

A F West, Child and Adolescent Mental Health Service, Wokingham Hospital, Wokingham, Berkshire RG4 1 2RE, UK

R R West, Epidemiology, University of Wales College of Medicine, Cardiff, UK

Correspondence to: Dr Andrew West

\section{REFERENCES}

1 Sackett DL, Haynes RB, Guyatt GH, et al. Clinical epidemiology: a basic science for clinical medicine. Boston: Little Brown, 1991.

2 Lemboke PA. Evolution of medical audit. JAMA 1967;199:111-8.

3 McLachlan G, ed. A question of quality. London: Oxford University Press, 1976.

4 West RR. Evidence based medicine, overviews, bulletins, guidelines and the new consensus. Postgrad Med J 2000;76:383-9.

5 Oxman A. Preparing and maintaining systematic reviews. In: Sackett D, ed. Cochrane Collaboration Handbook. Oxford: NHS R\&D, 1994

6 Black $D$. The limitations of evidence. J R Coll Physicians Lond 1998;32:23-6.

7 Surgeon General. Health consequences of smoking: cancer. Rochville, MD: US Department of Health, Education and Welfare, 1982.

8 Gehlbach SH. Interpreting the medical literature: a clinician's guide. Lexington: Collamore Press, 1982. 
9 Thompson SG, Pocock SJ. Can meta-analysis be trusted? Lancet 1991;338:1 127-30.

10 Anonymous. Meta-analysis under scrutiny [editorial]. Lancet 1997;350:675.

11 Naylor CD. Meta-analysis and meta-epidemiology of clinical research. $B M$ 1997:315:617-9.

12 Katerndahl DA, Lawler WR. Variability in meta-analytic results concerning the value of cholesterol reduction in coronary heart disease: a meta-meta-analysis. Am J Epidemiol $1999 ; 149: 429-41$
13 Hopayian K. The need for caution in interpreting high quality systematic reviews. BM 2001;323:681-4.

14 Pisek PE, Greenhalgh T. The challenge of complexity in health care. $B M$ 2001;323:625-8.

15 Broadhurst PL. The interaction of task difficulty and motivation: the Yerkes-Dodson Law revisited. Acta Psychol 1959;16:321-38.

16 Wickramasekera I. A conditioned response model of the placebo effect. In: White $L$ Tursky B, Schwartz GE, eds. Placebo: theory, research and mechanisms. New York: Guilford Press, 1985: 255-87.

17 Andrews G. Placebo response in depression bane of research, boon to therapy. $\mathrm{Br} J$ Psychiatry 2001;178:192-4.

18 Lyth IM. The functioning of social systems as a defence against anxiety. In: Lyth IM, ed Containing anxiety in institutions. Selected essays. Vol 1. London: Free Association Books, 1988: 43-85.

19 Heisenberg W. Unbestimmtheit. Zeits f Physik 1927;43:172.

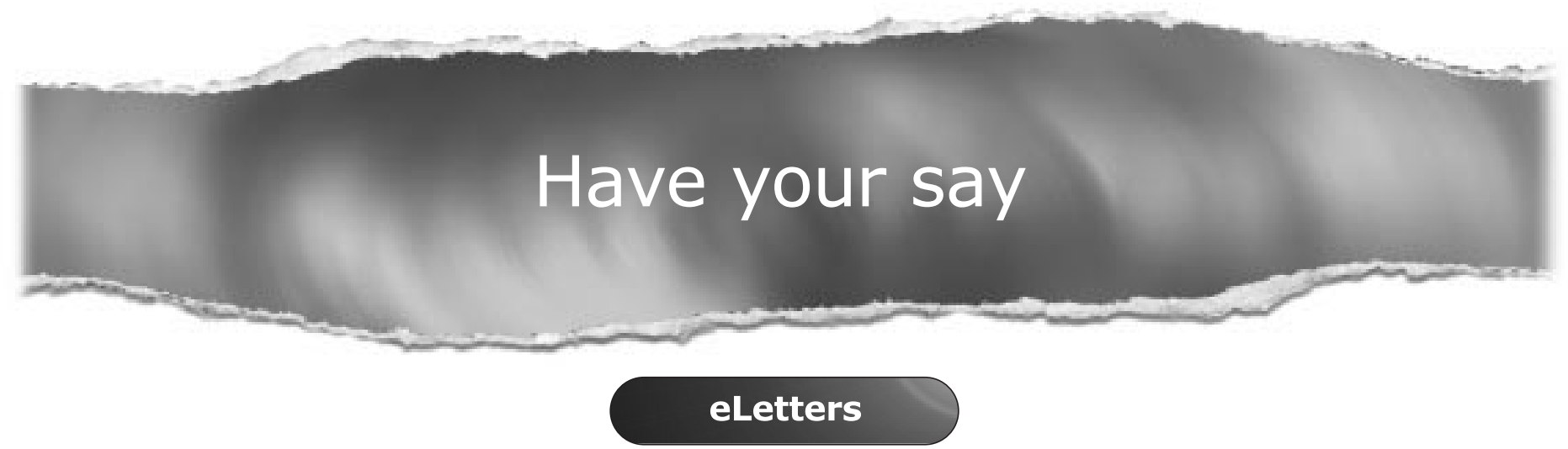

If you wish to comment on any article published in the Postgraduate Medical Journal you can send an eLetter using the eLetters link at the beginning of each article. Your response will be posted on Postgraduate Medical Journal online within a few days of receipt (subject to editorial screening).

www.postgradmedj.com 